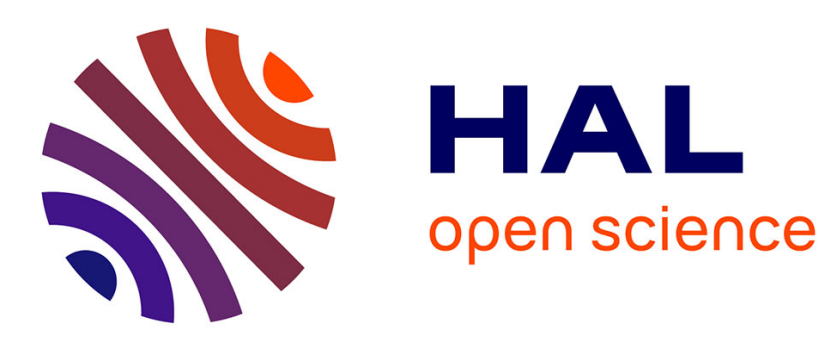

\title{
LINE SHAPES OF HYDROGEN AND HYDROGENIC IONS IN PLASMAS
}

\author{
C. Stehle
}

\section{To cite this version:}

C. Stehle. LINE SHAPES OF HYDROGEN AND HYDROGENIC IONS IN PLASMAS. Journal de Physique IV Proceedings, 1991, 01 (C1), pp.C1-121-C1-132. 10.1051/.jp4:1991115 . jpa-00249753

HAL Id: jpa-00249753

https://hal.science/jpa-00249753

Submitted on 1 Jan 1991

HAL is a multi-disciplinary open access archive for the deposit and dissemination of scientific research documents, whether they are published or not. The documents may come from teaching and research institutions in France or abroad, or from public or private research centers.
L'archive ouverte pluridisciplinaire HAL, est destinée au dépôt et à la diffusion de documents scientifiques de niveau recherche, publiés ou non, émanant des établissements d'enseignement et de recherche français ou étrangers, des laboratoires publics ou privés. 


\section{LINE SHAPES OF HYDROGEN AND HYDROGENIC IONS IN PLASMAS}

\section{STEHLE}

DAMAP et URA 812 du CNRS, Observatoire de Paris, Section de Meudon, 5. Place Jules Janssen, F-92195 Meudon Cedex, France

Résumé: les profils des raies de Lyman de l'hydrogène et des ions hydrogénoïdes sont présentés,en utifisant le formalisme du
Microchamp Modèle. Les notions de régimes collisionnel ("basses densités") et de limites statique ("hautes densités") sont analysées, ainsi que les conditions requises pour l'application de la limite de Holstmark dans les ailes.

Abstracts; Stark protiles of the Lyman lines of hydrogen and hydrogenic ions are presented here for weakly correlated plasmas, using the MMM theory. The notions of "low density" collisional and "high density" static limits are analysed, and also the conditions required for the use of the Holstmark theory in the wings.

Though the structure of hydrogenic ions is known with a very good accuracy, this is not the case for their line shapes in plasmas, where the results don't have a precision greater than $10 \%$ in the best cases. Discrepancies between the results of the various theories can even reach an order of magnitude. This is a consequence of the complex behaviour of the long range interaction between the hydrogenic emitter and the various plasma charges : simultaneous interactions can occur between them. Such effects are commonly called $\mathrm{N}$-body effects. As an exact analytical treatment is untractable, theoricists have to resort to simplifications, such as the static and impact approximations,leading to a soluble problem. Unfortunatly these methods are only asymptotical limits (section I). An other way is to use semi-empirical or numerical modelisations or simulations of the plasma-plasma and plasma-radiator interactions, leading to numerical tractable calculations and covering a larger domain of applicability. One of them is the Model Microfield Method (MMM), developped in (1971) by Brissaud and Frisch/1/, who pointed out, at first time, the influence of the ionic motions on the profile. This method uses an unusual modelisation of the time statistic of the plasmaemitter interactions (section II). However,MMM results are very close 10 those given by more "elaborate" approaches (for atomic physics point of view), as shown in section 111 for the Hydrogen and He+ Lyman lines. Among all the available methods, this last one,yielding satisfying profiles, is the less computer time consuming.As opacity calculations for astrophysical applications require, for hydrogen and also for hydrogenic ions , a large number of profiles in various different temperature and density conditions, it is necessary to develop such semi-empirical methods.

Up to now, this description is nevertheless restricted to the near line wings of weakly correlated plasmas ; the plasma charges can then be considered as independent particles, moving on rectilinear trajectories. The correlation effects are measured by the correlation parameter $\Gamma_{e}$, which is the rate of the mean Coulombic interaction potential,between two free plasma electrons, to the kinetic energy $\mathrm{kT}$ (table 1 ):

$$
\Gamma_{\mathrm{e}}=\frac{\mathrm{e}^{2}}{\mathrm{r}} \frac{1}{\mathrm{kT}}
$$

where $\bar{r}$ is the mean interelectronic distance defined by :

$$
\frac{4}{15}(2 \pi)^{3 / 2} N_{e^{-r^{3}}=1}
$$

\begin{tabular}{|c|c|c|c|}
\hline$T(K)$ & $10^{4}$ & $10^{7}$ & $10^{7}$ \\
\hline$N_{e}\left(\mathrm{~cm}^{-3}\right)$ & $10^{13}$ & $10^{18}$ & $10^{23}$ \\
\hline$\Gamma_{\mathrm{e}}$ & 0.007 & 0.0004 & 0.015 \\
\hline
\end{tabular}

Table 1

At large densities or low temperatures, the plasma is strongly correlated and only few semi-empirical recent methods such as the the microfield method of Boercker et al./2/ or refined Monte Carlo methods/3/ are actually available (in the line center). The MMM cannot yield correctly the core in that case, but could be used for the wings.

Let us point out that the assumption of low correlations supposes also that, at same typical distance $\overline{\mathbf{r}}$ from the hydrogenic radiator( of total charge $\left(Z_{e}-1\right)$ ), the plasma charges(electrons and ions of charge $Z_{p}$ ) don't deviate greatly. Hence the Coulombic attractive $\left(Z_{e}-1\right) e^{2 / \bar{r}}$ or repulsive $\left(Z_{e}-1\right) Z_{p} e^{2 / \bar{r}}$ potential remains smaller than $\mathrm{kT}$. 
Finally we will suppose that the atomic structure of the radiating ion is not modified by the plasma ; the number of free electrons inside the orbit of the bound electron must be small,i.e.:

$$
\overline{\mathrm{r}}>>\frac{a_{0}(\mathrm{n}-1)}{Z_{\mathrm{e}}}
$$

\section{I - THE LIMITING CASES}

Hereafter we shall consider a weakly correlated plasma composed by electrons (density $N_{e}$,temperature $T$ ), ions (density $N_{p}$,total charge $Z_{p}$,temperature $T$ ) and same traces of hydrogenic radiating ions (nuclear charge $Z_{\theta}$,temperature $T$ ).

The long range dipolar potential of interaction between the radiator and one plasma charge (section (I-1) is, in fact,screened at large distances from the radiator by the various other free charges of the plasma lying between the radiator and this charge, leading to the Debye Hückel potential,for the "binary" electronradiator interaction;the screening length is the Debye length $\lambda_{D}$ defined by:

$$
\lambda_{\mathrm{D}}{ }^{2}=\frac{\mathrm{k} \mathrm{T}_{\mathrm{D}}}{4 \pi \mathrm{e}^{2} \mathrm{~N}_{\mathrm{e}}}
$$

The interaction between a rapidly moving plasma electron and the radiator is screened by the other plasma electrons. The interaction between a slowly moving plasma ion and the radiator is screened by the other free ions and electrons so that :

$$
\begin{array}{lr}
T_{D}=T & \text { for the electrons } \\
T_{D}=T /\left(1+Z_{p}\right) & \text { for the ions }
\end{array}
$$

This model supposes a large number of particles inside the Debye sphere $\left(\lambda_{D}>>\bar{r}\right)$, wich is equivalent to $\Gamma_{\theta}<<1$.

1-1/ Time scales:Stark broadening results from the fact that the emitter simultaneously radiates at the frequency $\omega$ and interacts with the other plasma particles. The radiator motions lead to the usual Doppler broadening,wich is taken into account via the usual convolution procedures.For the line broadening at the detuning $\Delta \omega$ from the line center, we can define two different times of interest : the first one,

$$
\tau(\omega)=|\Delta \omega|^{-1}
$$

is a "pure radiative" time scale for the emission without plasma. The second one is the typical duration $\tau_{\mathrm{c}}$ of an elementary radiationless interaction process between the radiator and the plasma.For the most frequent low interactions (with perturbers at large distances) this time is:

$$
\tau_{c}=\frac{\lambda_{D}}{\bar{v}}=\omega_{p}{ }^{-1}
$$

where $\bar{v}$ is the thermal velocity, $\omega_{p}$ the plasma frequency.It represents the time for one particule to crossover the Debye sphere. As electrons and ions move with different velocities, there are two plasma frequencies $\omega_{p e}$ and $\omega_{p i}$ (electronic and ionic). The duration of a strong interaction however, depends on the strength of this interaction. For example if this interaction is attributed to a charge of velocity $v$ at a very small distance $r$ from the emitter, this time is :

$$
\tau_{c}=r / v
$$

The comparison between the radiative and plasma times of interest justifies different descriptions for the broadening.

In this section, we shall consider, separatively the ionic or the electronic contribution to the intensity and discuss the corresponding limiting cases, for the $n \rightarrow n^{\prime}\left(n>n^{\prime}\right)$ transitions.

1-2/ Line center:For detunings of the order of magnitude of the line width $\Delta \omega_{1 / 2}$, these two times are $\Delta \omega_{1 / 2}{ }^{-1}$ and $\omega_{p}{ }^{-1}$.

a) At low densities, the halfwidth is small, thus :

$$
\Delta \omega_{1 / 2}<<\omega_{p}
$$

This is the usual validity condition of the collisional approach,requiring that the mean time interval $\Delta T$ between two collisions is much larger than the typical collisional duration $\tau_{c}=\omega_{p}{ }^{-1} ; \Delta T$ is the inverse of the 
collision rate and is equal to $\langle N \sigma v\rangle^{-1}$ i.e $\Delta \omega_{1 / 2}{ }^{-1},(\sigma$, collisional cross section of interest for the broadening). Thus, the inequality $\Delta T=\Delta \omega_{1 / 2}{ }^{-1} \gg \tau_{c}=\omega_{p}{ }^{-1}$ is equivalent to (9).

In a semi classical perturbational calculation, the electronic and ionic contributions to the halfwidth have analytical expressions $/ 4,5 /$; the quantity $\Delta \omega_{1 / 2} \omega_{p}^{-1}$ appearing in (9) varies as :

$$
\Delta \omega_{1 / 2} \omega_{p}^{-1} \sim k_{n n^{\prime}} \sqrt{\frac{N_{e}}{k T\left(1+Z_{p}\right)}} \frac{Z_{p}}{Z_{e}^{2}} \frac{\hbar^{2}}{m_{e}{ }^{2}}
$$

$m$ is the reduced mass of the colliding pair (radiator+perturber) and $k_{n n}$ is a number depending on the (nn') transition and increasing with $n$.

If this inequality is satisfied, the collisional formalism is justified for the whole profile from the center up to the wings. In the line center, this inequality corresponds to the usual impact approximation, and the shape is Lorentzian/4/:

$$
I(\omega) \approx \frac{1}{\pi} \frac{1}{\Delta \omega+i \Delta \omega_{1 / 2}}
$$

Let us point out that the impact limit is obtained at low $N, m, Z_{p}$ and large $T, Z_{e}$ values. Hence the so called "low density" and "high density" limits will be heareafter referred to the condition (9). They are,in fact,dependent on the line $\left(n, n^{\prime}\right)$ and on the plasma conditions .

b) Intermediate densities : for higher densities, the halfwidth is larger and :

$$
\Delta \omega_{1 / 2} \geq \omega_{p}
$$

In terms of collisions, this indicates the occurence of time overlapping long range interactions. These simultaneous weak interactions between the emitter and the plasma charges reflect the invalidity of the collisional treatment for the broadening in the line center. Let us however notice,that the shorter strong interactions are still well separated in time for sufficient small $\left(r<<\lambda_{D}\right)$ distances of interest. As the intensity in the line wings is mainly concerned with these short range interactions, the line wings can even be described within the frame of the binary collisional approach although this is not the case for the line core, where we are interested into all the interactions, especially the long range ones, which are the most frequent.

c) High density plasma : the radiative time of interest is negligible compared to the typical time $\omega_{\mathrm{p}}{ }^{-1}$ for the plasma ,thus:

$$
\Delta \omega_{1 / 2} \gg \omega_{p}
$$

In this case, a static formulation of the broadening is justified, leading to the following expression for the intensity :

$$
I(\omega)=\int I\left(\omega ; \vec{r}_{1} \ldots \vec{r}_{n}\right)^{2} \overrightarrow{d r}_{1} \ldots \vec{d}_{r_{n}} P\left(\vec{r}_{1} \ldots \vec{r}_{n}\right)
$$

where $\mathrm{I}\left(\omega ; \overrightarrow{\mathrm{r}}_{1} \ldots \overrightarrow{\mathrm{r}}_{\mathrm{n}}\right)$ is the intensity, radiated by $\mathrm{n}$ fixed plasma charges at the distances $\overrightarrow{\mathrm{r}}_{1}, \ldots, \overrightarrow{\mathrm{r}}_{\mathrm{n}}$ from the radiator. $P\left(\vec{r}_{1} \ldots \vec{r}_{n}\right)$ is the probability of this spatial configuration.

As the linewidth increases with increasing $n, n^{\prime}, N, Z_{p}$, or decreasing $T, Z_{e}$, similar variations are expected for the definition of the static limit from (13)

$\mathrm{I}-3 /$ Line wings :The radiative time scale is now $\tau(\omega)=|\Delta \omega|^{-1}$ which is much smaller than $\Delta \omega_{1 / 2}{ }^{-1}$

$$
|\Delta \omega| \gg \Delta \omega_{1 / 2}
$$

a) low density plasmas (condition 9):the typical times are $\Delta T=\Delta \omega_{1 / 2}{ }^{-1}$, time interval between two collisions of typical duration $\tau_{c}$ and $|\Delta \omega|^{-1}$. The small value of $|\Delta \omega|^{-1}$ compared to $\Delta T$ allows to select only one collision for the radiative process. The total intensity is the average of the collisional contributions

$$
I(\omega)=N\left\langle\sum_{\mathrm{i}, \mathrm{f}} \mathrm{d}_{\mathrm{if}}{ }^{2} \delta\left(\hbar \omega-E_{\mathrm{f}}+E_{\mathrm{i}}\right)\right\rangle_{\text {Average }}
$$

where $i$ and $f$, (with respective energies $E_{i}, E_{f}$ ) correspond to the eigenstates of the colliding pair (radiator +perturbing charge). This is the one perturber approximation $/ 6 /$.

At intermediate detunings, i.e., if $|\Delta \omega|^{-1} \geq \omega_{i}^{-1}\left(\omega_{i}^{-1}\right.$ typical time duration of the distant,long range 
interactions), all the collisions contribute to the intensity (16), especially the distant ones which are the most frequent. The shape is sensitive to "wholeness" of each collision. But at larger detunings the intensity will be sensitive to portions of the collisions, leading to more reduced velocity effects on this small time scale. The principal contribution comes from distance ranges around the value $r_{\omega}$ satisfying the relation $h_{\Delta \omega}=V_{f}\left(r_{\omega}\right)-V_{i}\left(r_{\omega}\right)$ where $V_{f}$ and $V_{i}$ are the potentials of interaction for a perturber at the fixed value $r_{\omega}$ from the radiator. Hence for large detunings, the dominant contribution will come from strong collisions, which only are able to satisfy this relation. These collisions do not overlap in time.

b) higher density-plasmas : For large values of the detuning,we are looking again inside non time-overlapping strong interactions. As explained before in $\$ 1.2 \mathrm{~b}$ the intensity in the wing can be described within the frame of the one perturber approach, although the collisional formalism is invalid in the center (overlapping weak interactions).

1 4/ The Holsmark limit: In the wings the radiative time of interest $\tau(\omega)$ can be very small,compared to the typical collisional duration of the relevant strong collisions, i.e.

$$
\tau(\omega)=|\Delta \omega|^{-1}<<\frac{r_{\omega}}{\bar{v}}
$$

The static limit is again valid in the frame of the binary (one perturber) model. The intensity is given by (16) where $E_{f}$ and $E_{i}$ are now the adiabatic eigen-energies at the fixed distance $r_{\omega}$. As the dipolar potential of interaction can be expressed in terms of the static electric field $e Z_{p} / r_{\omega}{ }^{2}(I I-1)$, one has :

$$
\hbar \omega-\left(E_{f}-E_{i}\right)=\hbar \Delta \omega-\left(C_{f}-C_{i}\right) \frac{e^{2} a_{o} Z_{p}}{r_{\omega}{ }^{2} Z_{e}}
$$

where $C_{f}, C_{i}$ are constants, associated to the linear Stark energy shifts for the diagonal parabolic eigenstates i $>, \mid$ i $>$. The average has to be taken, here, according to the probability to find the charge $Z_{p}$ at the distance $r_{\omega}$. One obtains the Holtsmark limit:

$$
I_{H}(\omega)-\Delta \omega^{-5 / 2} Z_{\theta} \cdot 3 / 2\left(\hbar / m_{\theta}\right)^{3 / 2} N_{\theta} Z_{p}^{1 / 2}
$$

Expressions (16) and (18) give the value of $r_{\omega}$ :

$$
r_{\omega} \sim \sqrt{\frac{\hbar Z_{p}\left(C_{f}-C_{i}\right)}{m_{e} Z_{e} \Delta \omega}}
$$

The validity conditions for the static wing formulation for the (nn') line are then (taking as lower estimate $\left(C_{f}-C_{j}\right)=n$, and using $\left.(17)\right)$ :

$$
\begin{aligned}
& \Delta \omega \gg \Delta \omega_{1 / 2} \text { and, } \\
& \hbar|\Delta \omega|>>k T \frac{Z_{e}}{Z_{p}} \frac{m_{e}}{m} \frac{2}{3 n}
\end{aligned}
$$

This limit is obtained at $\omega_{\mathrm{Hi}}$ for the ions and at $\omega_{\mathrm{He}}=\omega_{\mathrm{Hi}} \mathrm{m} / \mathrm{m}_{e}$ for the electrons. Between $\omega_{\mathrm{Hi}}$ and $\omega_{\mathrm{He}}$, the electronic intensity deviates from the static limit. This is attributed to electronic dynamics effects. Beyond $\omega_{H e}$, the intensity $I(\omega)$ equals $2 I_{H}(\omega)$.

The Holtsmark limit corresponds to the static limit of the one perturber approach in its semi-classical description with rectilinear trajectories and dipolar interaction. In the hydrogenic case deflexion (or correlation leffects are negligible at $r_{\omega}$ if :

$$
\frac{\mathrm{Z}_{\mathrm{p}}\left(\mathrm{Z}_{\left.\mathrm{e}^{-1}\right) \mathrm{e}^{2}}\right.}{\mathrm{r}_{\omega}}<\mathrm{kT}
$$

Equations 20,22 , combined with the most pessimist case $\left(C_{f}-C_{j} \approx 3 n / 2\right)$ yield:

$$
\Delta \omega\left(Z_{e^{-1}}\right)^{2}<\frac{3 n(k T)^{2} \hbar}{2 m_{e} e^{4} Z_{p} Z_{e}}
$$

Combining again with (21), one finds for the $\left(n n^{\prime}\right)$ line : 


$$
\mathrm{kT}>\mathrm{Z}_{\mathrm{e}}^{2}\left(\mathrm{Z}_{\mathrm{e}}-1\right)^{2} \frac{4 \mathrm{~m}_{\mathrm{e}}{ }^{2} \mathrm{e}^{4}}{9 \mathrm{~h}^{2} \mathrm{~m} \mathrm{n}^{2}}
$$

To summerise,the usual Holtsmark limit is easier reached in the wings for the ions than for the electrons. In the later case, departures from the dipolar interaction, (quantum, exchange, quadrupolar, polarisation effects ...) can be large at $r_{\omega}$ defined by (20) and (21). This point has to be carefully analysed and is out of the subject of the present communication,which is restricted to the case of the hydrogen and helium lines for weakly correlated plasmas, and in the dipolar approach

15/Resume : The preceding remarks were devoted to one species of perturbers (i.e. the electrons or the ions). Usually the rapidly moving electrons can be described within the collisional approach, because (9) is always satisfied except,probably,for the highest lines.The situation is not so clear for the slow ions where (9)is satisfyied only for the so called "low density plasmas." The usual static description for the ionic contribution to the intensity is suitable for high density plasmas or high lines $\left(n>>n^{\prime}\right)$ or low temperatures. In the line wings all these approaches converge to the one perturber approximation, leading for the intensity to additive ionic and electronic contributions.

The line cores and near wings are governed by the dipolar interaction potential. In this case,the large involved distances of interaction between the radiator and the perturbers legitimate the use of rectilinear trajectories and of a semi-classical description for the relative motions. These three approximations are simultaneously assumed to obtain the usual $\Delta \omega^{-5 / 2}$ static limit for electrons and (or)ions. We have shown that this Holtsmark limit is, in these conditions, reached at smaller detunings for the ions than for the electrons. We will put emphasis again on the fact that,the breakdown of only one of these 3 conditions at large detunings prevents the use of the Holtsmark limit in the far wings.

16/ The different approaches: Among the various descriptions of the Stark broadening, the most frequent ones have been devoted to the line cores using an impact treatment for the electrons and a static description for the ions. More elaborate treatments are the "unified theories" with larger domain of validity.

a) unified theories for the electrons : in this case the electronic broadening is "correctly" given from the core to the far line wings ; the more accurate methods use a quantum description of the relative motion with a correct description of the short range effects (Lyman $\alpha$ of hydrogen, $/ 7,18 /)$ or a dipolar interaction $\left(\mathrm{H}_{\beta}\right.$ of hydrogen $/ 8 /$, hydrogenic ions lines $/ 9 /$ ). Other approaches use a semi classical description of the electronic broadening in the dipolar /10-14/ or with more accurate potentials /15/.All these calculations use a static description for the ionic broadening.

b) uniffed low density theories for electrons and ions : in the case of Lyman $\alpha$ of hydrogen, an exact dipolar calculation has been done for the ionic and electronic contributions to the broadening/16/.

c) semi empirical methods : as long as the electrons can be described in a semi-classical approach, simulation methods can be used. This has been done for the low hydrogen lines and for the line center for the ionic perturbers with impact electrons $/ 17 /$.Other approaches use a modelisation of the plasma statistics, as the Model Microfield Method /1,2,19/.The advantages of the MMM are,first to give a practical interpolation method between the low (9) and high (13) density limits and between the low density impact core and the static wing, and secondly to be very easy to use, requiring non prohibitive computer times. The results presented here,using the MMM,are in good agreement with those given by more elaborate theories.

\section{II - LINE SHAPES IN THE MMM METHOD}

As the Model Microfield Method uses a description of the plasma radiator in terms of electric microfield $\overrightarrow{\mathrm{E}}$, we shall first introduce this notion and formulate again the limiting impact and static cases in terms of microfields, then describe the MMM and give the results.

$111 /$ Field notion: Let us introduce firstly the interaction between one perturber of charge $Z_{p}$, at the distance $r$ from the nucleus (charge $Z_{e}$ ) of an hydrogen atom or an hydrogenic ion with a bound electron at the distance $r_{s}$ from the nucleus. The potential of interaction is, neglecting the Debye screening,

$$
V\left(\vec{r}, \vec{r}_{s}\right)=-\frac{z_{p} e^{2}}{\left|\overrightarrow{r_{s}}-\vec{r}\right|} \frac{z_{p} z_{e} e^{2}}{|\vec{r}|}
$$

which vields for $r \gg r_{s}$ : 


$$
\mathrm{V}\left(\overrightarrow{\left.\mathrm{r}, \vec{r}_{\mathrm{s}}\right)}=\frac{\mathrm{Z}_{\mathrm{p}}\left(\mathrm{Z}_{\mathrm{e}}-1\right) \mathrm{e}^{2}}{|\overrightarrow{\mathrm{r}}|}-\overrightarrow{\mathrm{d}}_{\mathrm{s}} \vec{\xi}\right.
$$

The first term (long range contribution of the monopole term)is the usual Coulombic interaction responsible of trajectories deflexions for ionic systems. The second term is the dipolar term, where $\left(\overrightarrow{\mathrm{d}}_{\mathrm{s}}=\mathrm{e} \overrightarrow{\mathrm{r}}_{\mathrm{s}}\right)$ is the electronic dipole, $\vec{\xi}$ the value of the electric microfield generated by the charge $Z_{p}$ on the radiator at distance $\mathrm{r}$ :

$$
|\vec{\xi}|=\frac{Z_{p} \mathrm{e}}{r^{2}}
$$

Higher order terms are neglected in the dipolar approximation. The total plasma radiator interaction is then :

$$
V=-\vec{d}_{s} \vec{E} \quad, \quad \vec{E}=\sum_{\text {charges } i} \vec{\xi}_{\mathrm{l}}
$$

where the total electric microfield, $\overrightarrow{\mathrm{E}}$, is the sum of a rapidly varying electronic contribution $\overrightarrow{\mathrm{E}}_{\mathrm{e}}$ and a more slowly varying ionic contributions $\vec{E}_{j}$ (resp. high frequency and low frequency contributions).Typical scales associated with their time fluctuations are respectively the inverse of the electronic and of the ionic plasma frequencies:

$$
\omega_{p i}=\frac{\lambda_{D i}}{v}=\omega_{p e} \sqrt{\frac{m_{e}}{m}}
$$

The electric microfield constitutes a randomly time dependent process.

II 2/ Static and impact limits : for simplicity,we restrict ourself to the case of one of the two (ionic or electronic) contributions to the profile.

a) Static limit : in the line center at high densities (13) or in the line wings (15),the static intensity is given by:

$$
I(\omega)-\sum_{i f} \int_{0}^{\infty} P(E) d E d_{i f}(E)^{2} \delta\left(\hbar \Delta \omega-C_{i f} E\right)
$$

where $|i>| f>$, are the usual parabolic eigenstates of hydrogenic ions perturbed by the static electric field $E$ parallel to the $z$ axis; $d_{i t}(E)$ is the corresponding dipolar element of transition between $i$ and $f_{;} C_{i} E$ (resp. $C_{f} E$ ) are the linear energy shifts, $C_{i f}=C_{i}-C_{f}$, and $\Delta \omega=\omega-\omega_{0}$ is the detuning from the line center. The relevant statistical electric field property is here the electric field distribution $P(E)$.

b) Impact limit : In the line center at low density (9),the quantity of interest is the relaxation rate $\gamma$, whose expression is, in a perturbational second order treatment proportionnal to:

$$
\left\{\int_{-\infty}^{+\infty} d t_{1} \int_{-\infty}^{t_{2}} V\left(t_{1}\right) V\left(t_{2}\right) d t_{2}\right\}_{\text {Average }}
$$

where $V(t)$ is the collisional dipolar potential of interaction (26) and the average is to be taken over all the collisions. One can shown that:

$$
\gamma-\int_{0}^{\infty} \Gamma(\mathrm{t}) \mathrm{dt}
$$

where $\Gamma(t)$ is the field covariance. Near the impact core, it can be shown that the relevant quantity is the Fourrier transform at $\Delta \omega$ of $\Gamma(t)=\langle\vec{E}(0) \vec{E}(t)\rangle ; \Gamma(t)$ is thus the quantity of interest for the broadening.Its expression is known for the case of weakly correlated plasma (rectilinear trajectories) $11 /$. 
III 3/ The Model Microfield Method modelises the statistical time properties of the electric microfield $\overrightarrow{\mathrm{E}}$. The field value is assumed to be constant by steps. The jumping times follow a Poisson law with the frequency $v(E)$, which is fitted to yield correctly the field covariance :

$$
\Gamma(t)=\int_{0}^{\infty} P(E) E^{2} \exp (-v(E) t) d E
$$

The intensity has an analytical expression in terms of $P(E), v(E)$ and $T_{S}(z, E)$, where $T_{S}(z, E)$ is the Laplace transform at $z=\omega+i v(E)$ of the time evolution operator $T_{s}(t)$ for the radiator perturbed by the static field $E$. This expression yields the static limit for $v(E)<<|\Delta \omega|$ and the impact limit in the opposite case(\$II.2). Hence, both of these two limiting cases are correctly obtained, as long as one uses the correct microfield distribution and covariance. The MMM gives an interpolation method between these opposite descriptions.It is thus an unified formalism, wich is, contrarily to the usual unified theories, not restricted to the "low-density" (\$l.2a) collisionnal limit for the electrons (\$l.6a) and/or the ions (\$l.6b).Thus,using appropriate field probabilities, it describes also correctly the overlapping strong collisions leading to the intermediate and high densities limits of $\S 1.2 b$,c. Let us note also that the static ion limit can be obtained in the frame of the MMM, just by taking $v(E)$ equal to 0 .

The following results for hydrogen an hydrogenic ions have been obtained, using an "average" electronic frequency-dependent operator.this approximation will be discussed in a subsequent paper.

\section{4/ Results for hydrogen}

a) Low density case : we first compare our results for Ly $\alpha$ with those given by the collisional theories in the low density case .For this line, at $\mathrm{T}=10^{4} \mathrm{~K}$ and in an hydrogen plasma, the validity condition (9) yields $\mathrm{N} \ll 10^{15} \mathrm{~cm}^{-3}$. At $\mathrm{N} \ll 10^{13} \mathrm{~cm}^{-3}$, both ions and electrons can be described within the collisional formulation leading to an analytical expression for the width $/ 16 /$. In the line center the electronic contribution is very small.

The width obtained using static ions is smaller of about one order of magnitude as shown on fig 1.The static ion profile converges to the MMM result in the wings. Hence, the collisional model gives the Holtsmark static limit (condition 21) for detunings larger than $0.06 \AA$ for protons and $55 \AA$ for electrons (Fig. 2). It is clear that,at this detuning, the short range and quantum effects for electrons prevent the use of a semi-classical dipolar approach with rectilinear trajectories; thus the static limit is strictly impossible to reach for the electrons $17,20 /$. As the line widths are very small compared to the Doppler width, both methods (static and dynamic ions) yield the same result after convolution with the Doppler effect.

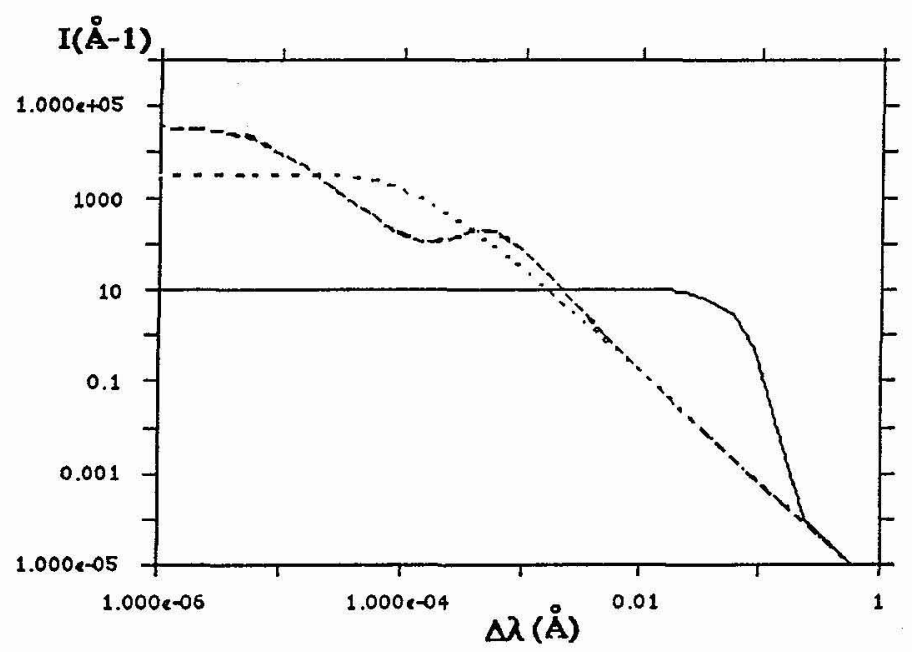

Figure 1:Lyman $\alpha$ line of hydrogen perturbed by protons and electrons $\left(\mathrm{N}_{e}=10^{13} \mathrm{~cm}^{-3}, T=10^{4} \mathrm{~K}\right)$; _. : dynamic ions , .-. static ions ,full line: after Doppler convolution (both static and dynamic limit are undistinguishable); The detunings corresponding to the electronic and ionic plasma frequencies are equal respectively to $1.410^{-2} \AA$ and $4.610^{-4} \AA$. 
Calculations of Feautrier et al. /18/ including quadrupolar, polarisation and exchange effects show that, if the static limit seems to be reached between 5 and $10 \AA$ for electrons, it is only a coincidence,quantum and static results diverging rapidly after $10 \AA$. A comparison between collisional quantum dipolar descriptions (Exact Resonance Model:ERM/16/) and MMM results for protons and electrons contributions to the wings is done in figure 2. The widths in the center are slightly larger in the collisional formulation than in the MMM. The static limit for the ions is reached at $10^{-2} \AA$ for the MMM instead of $0.1 \AA$ for the ERM. But discrepancies between these two methods remain very small. A similar conclusion can be given for the electrons.

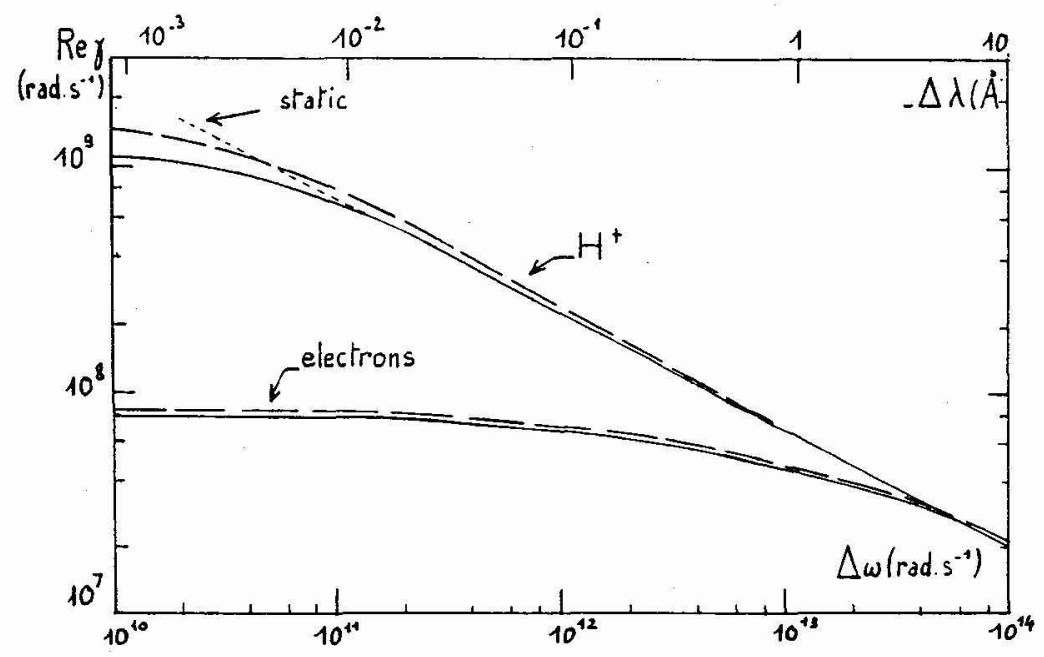

Figure 2: Lyman $\alpha$ line of hydrogen perturbed by protons and electrons $\left(10^{4} \mathrm{~K}, 10^{13} \mathrm{~cm}^{-3}\right)$. Variations with $\Delta \omega$ of the real part of the frequency dependent width $\gamma(\omega)$, defined by : $\pi \mathrm{I}(\omega)=\operatorname{Re}\left[(\Delta \omega+\gamma(\omega))^{-1}\right]$; this quantity is the sum of an ionic $\left(\mathrm{H}^{+}\right)$and an electronic con. tribution : $\gamma(\omega)=\gamma_{e}(\omega)+\gamma_{j}(\omega)$; full line:MMM results, _. ERM results, ..... static limit.

b) Intermediate densities :(condition 12) at $\mathrm{N}_{\mathrm{e}}=10^{17} \mathrm{~cm}^{-3}$ and $\mathrm{T}=10^{4} \mathrm{~K}$ for Lyman $\alpha$ (Figures $3 \mathrm{a}$ and b) the discrepancies between the results obtained assuming "exact" dynamic ions and static ions are smaller.But, due to the relative large contributions of the collisional broadening to the linewidth, the departures remain visible after Doppler convolution (fig. 3b).

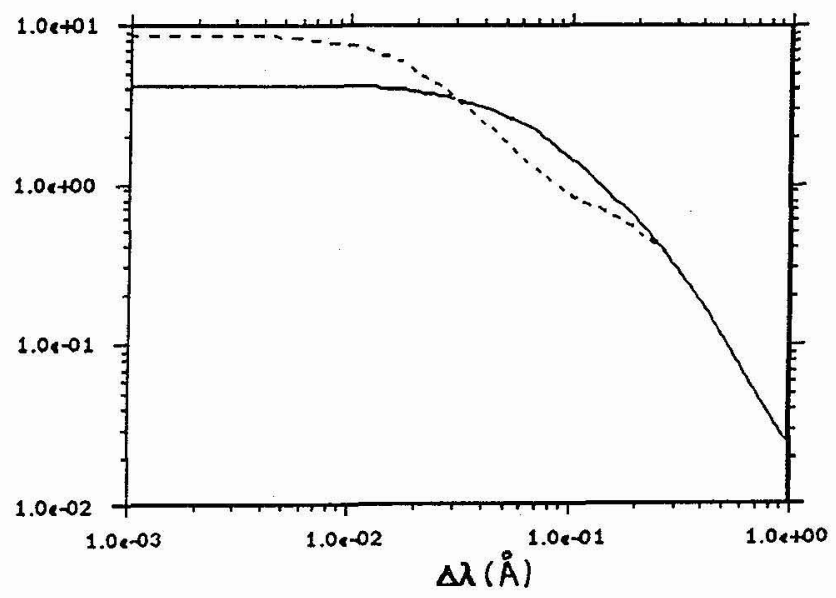

Fig. 3a 


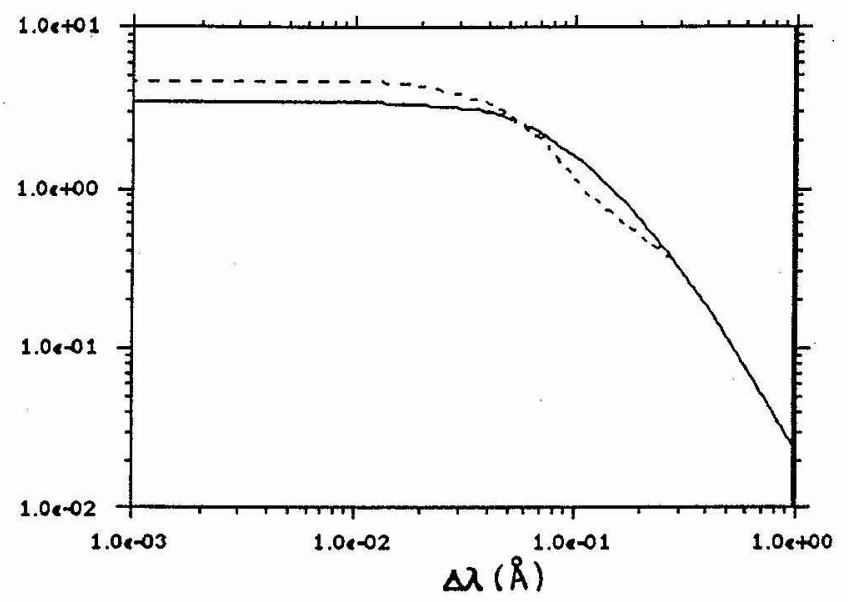

Fig $3 b$

Figures 3 a and $b$ : Lyman $\alpha$. line of hydrogen perturbed by protons and electrons $\left(T=10^{4} K, N_{e}=10^{17} \mathrm{~cm}^{-3}\right)$ before (a) and after(b) Doppler convolution.Full line:dynamic ions, --: static ions. The electronic and ionic plasma frequencies are $1.4 \AA$ and $4.610^{-2} \AA$.

c) Effects of the density on the halfwidth : we have shown that for a given value of $T$ and of the reduced mass $\mathrm{m}$, the ions are more static, as the density $\mathrm{N}$ increases. This is illustrated on figure 4 , where the halfwidth is in reduced units $\Delta \alpha_{1 / 2}=\Delta \lambda_{1 / 2} / F_{0}\left(F_{0}=1.2510^{-9} \mathrm{~N}^{2 / 3}\left(\mathrm{~N}\right.\right.$ in $\left.\mathrm{cm}^{-3}\right)$, is the Holtsmark field.). The plasma contains traces of hydrogen diluted in argon, at $10^{4} \mathrm{~K}$. As the density increases,static and dynamic results seem to converge slightly. MMM results are compared at low density to those obtained in the impact collisional formulation $/ 16 /$, which yields larger widths, as mentioned before (\$ll.4a). The agreement between MMM results and those obtained by $\mathrm{Oza}$ et al./20/ in the relaxation theory is correct over this density range.

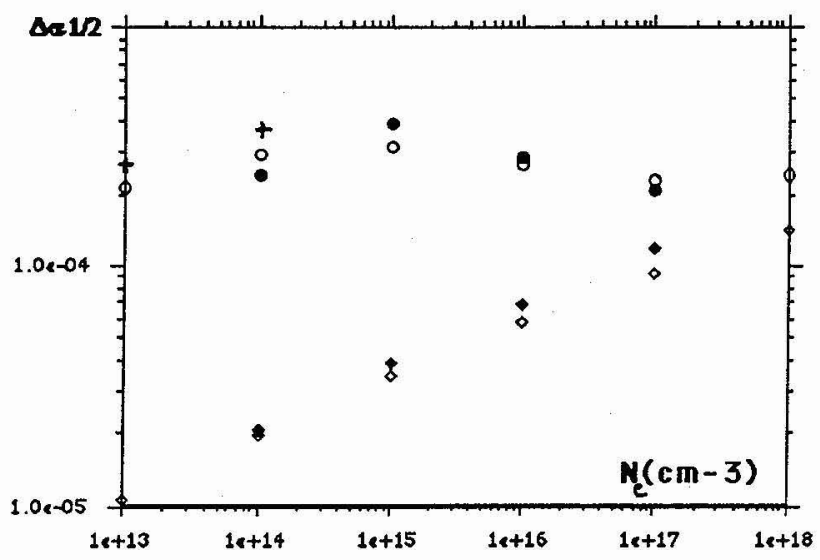

Figure 4 : halfwith of the Lyman $\alpha$ of Hydrogen perturbed by $\mathrm{Ar}^{+}$and electrons $\left(T=10^{4} \mathrm{~K}\right.$ ), versus the electronic density:Oza/20/ (static and dynamic • ions) ; MMM (static $\diamond$ and o dynamic ions); +: impact limit/4/ (ions and electrons).

d) Effects of the reduced mass : convergence to the static limit is also obtained for the ions when the reduced mass between the radiator and the perturbing ion increases, at fixed values of the density and temperature (figure 5). 


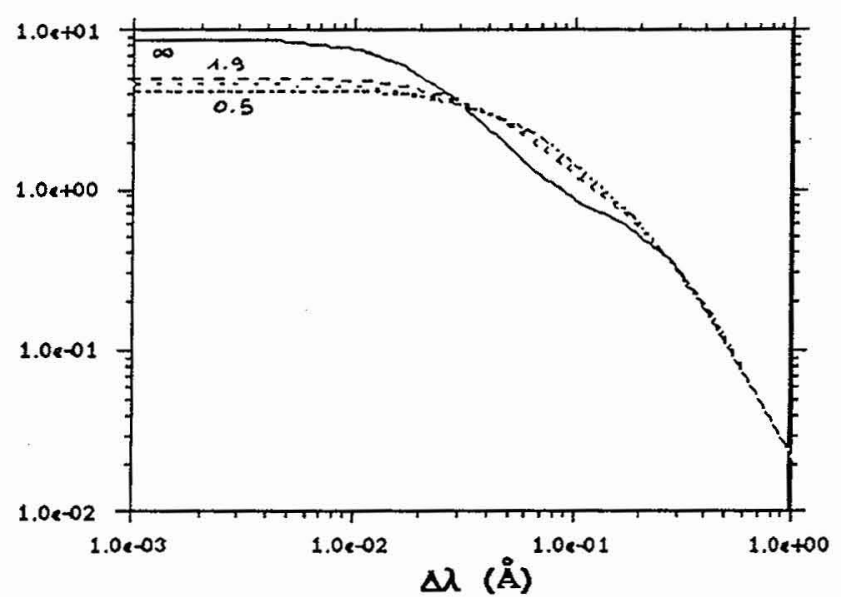

Figure 5: Lyman $\alpha$ of hydrogen and deuterium at $10^{17} \mathrm{~cm}^{-3}$ and $10^{4} \mathrm{~K}$ for different reduced masses $m=\infty, 1.90\left(\mathrm{D}+\mathrm{Ar}^{+}\right), 0.98\left(\mathrm{H}_{+} \mathrm{Ar}^{+}\right), 0.5\left(\mathrm{H}+\mathrm{H}^{+}\right)$.

e) Effects of the line : as mentioned in \$I.2 the notion of high density (i.e. leading to the static limit for the ions) depends also on the line. The present results for the Lyman 7 line at $10^{13} \mathrm{~cm}^{-3}$ show that in the absence of the Doppler effect, the ions are nearly static, although they are "impact" in the Ly $\alpha$ case (Figure 6).

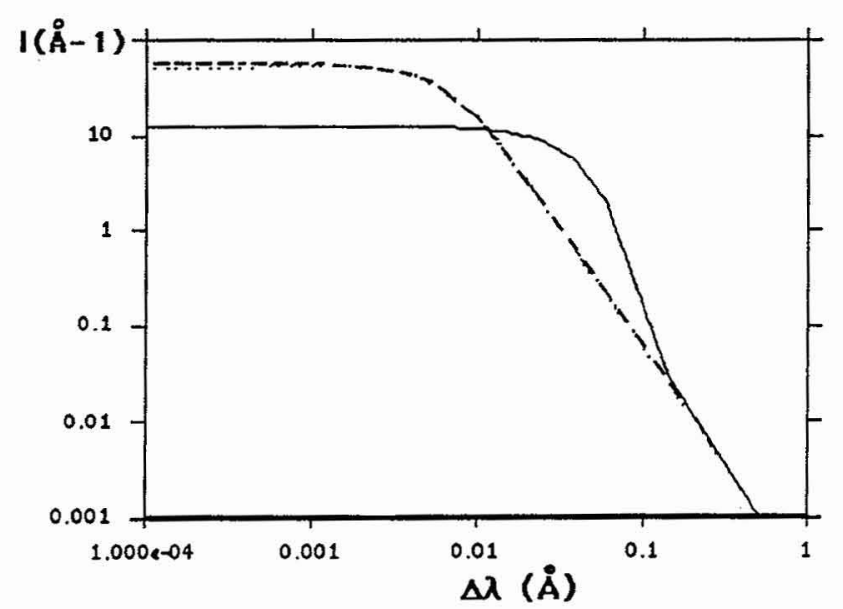

Figure 6 :Lyman 7 of Hydrogen $\left(n=7, n^{\prime}=1\right)$ perturbed by protons and electrons at $10^{17} \mathrm{~cm}^{-3}$ and $10^{4} \mathrm{~K}$; ,...:static ions,full line: after Doppler convolution (both static and dynamic limit are undistinguishable). :dynamic ions

II 5/ Results for the hydrogenic helium: as indicated in \$l.2a, the low density limit leading to a collisional treatment for the ionic broadening is easier to obtain for hydrogenic emitters than for hydrogen,owing to the larger $Z_{e}$ value. On the other hand, the reduced mass is slightly larger. In the case of $\mathrm{He}^{+}\left(\mathrm{Z}_{e}=2\right)$ perturbed by protons (reduced mass $m \sim 0.8$ ) and electrons, there are no large differences between the low and high density limits:the factor $\mathrm{m} / \mathrm{Z}_{\mathrm{e}}{ }^{2}$ in expression 10 is thus equal to 0.2 for $\mathrm{He}^{+}$instead of 0.5 for H.Ion dynamics effects are shown at $10^{18} \mathrm{~cm}^{-3}$ and $10^{5} \mathrm{~K}$ for Lyman $\alpha$ on figure 7 . 


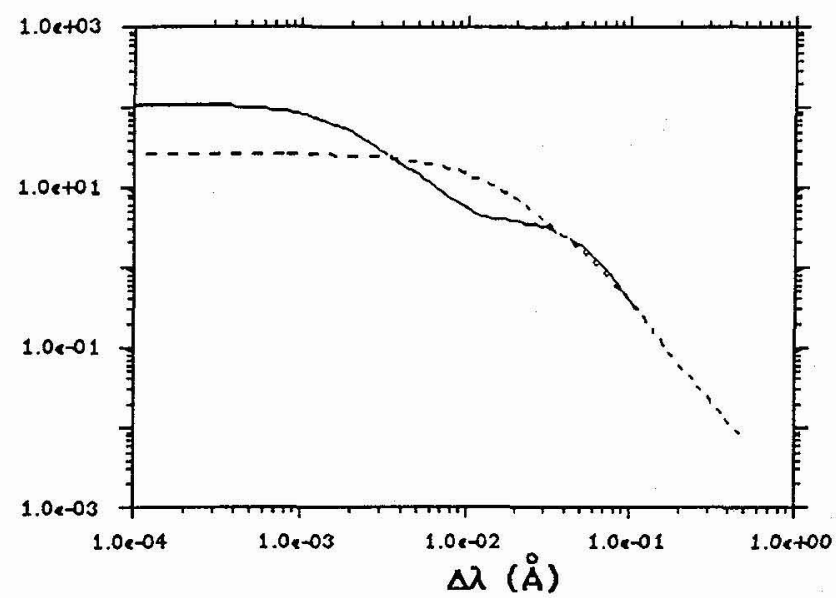

Fig.7a

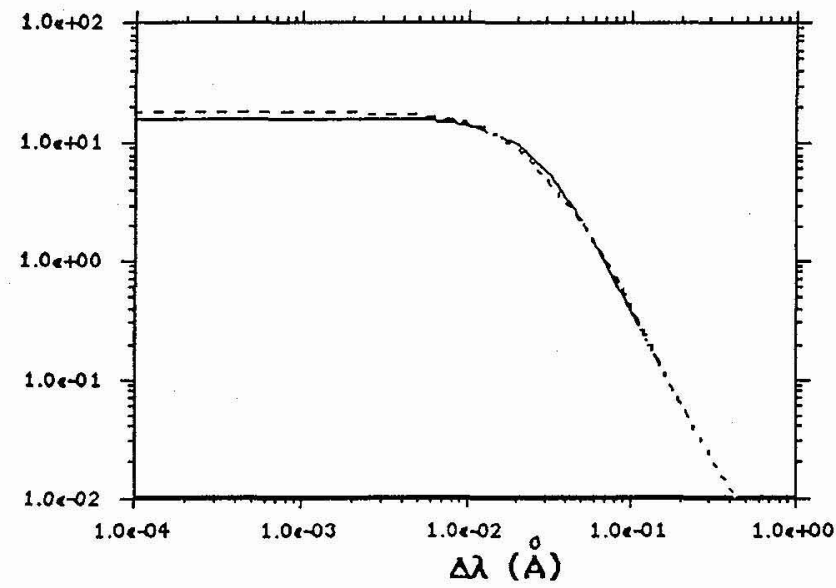

Figures $7 \mathrm{a}$ and $\mathrm{b}$ : Lyman $\alpha$ line of hydrogenic helium perturbed by protons and electrons $\left(T=10^{7} \mathrm{~K}, \mathrm{~N} \mathrm{e}_{\mathrm{e}}=10^{\mathrm{Fig}} .7 \mathrm{~b}\right.$ fore(a) and after(b) Doppler convolution. The electronic and ionic plasma frequencies correspond to $0.8210^{-2} \AA$ and 0.27 $10^{-3} \AA$.;---:dynamic ions, full line:static ions ;

\section{III - CONCLUSION}

Though the MMM is rough, it is reliable from the center to the wings and for all the densities. The comparison with the results obtained in other approaches is satisfying. This allows us to extend this method to weakly correlated plasmas for higher hydrogenic emitters. However let us point out that the applicability is restricted $1 /$ to the case where fine structure effects are negligible. These effects, increasing with $Z_{e}$, only affect the line cores ; 2/to temperatures and densities such as the plasma correlation effects are not too large $; 3 /$ to near line wings.

The results presented here indicate that in the astrophysical conditions of stellar atmospheres ( $\mathrm{N} \leq 10^{15} \mathrm{~cm}^{-3}, T \sim 10^{4} \mathrm{~K}$ ), the large Doppler effects tend to mask the effects of ion dynamics. Nevertheless, MMM yields good results for the electronic broadening as well. But the plasma condition of the stellar enveloppes are very different $\left(\mathrm{N} \sim 10^{22} \mathrm{~cm}^{-3, T} \sim 10^{6} \mathrm{~K}\right.$, highly charged hydrogenic emitters). It is actually impossible to predict whether these same conclusions can be drawn at these densities.

ACKNOWLEGMENTS: I am grateful to G. Nollez and A. Mazure for their assistance ,to N. Allard for taking part in some of this work, wich has been carried out in conjuction with N. Feautrier. 


\section{REFERENCES:}

11/ Frisch U. and Brissaud A. , J. Quant. Spectrosc. Radiat. Transfer ,11,1753

(1971),Brissaud A. and Frisch U. ,J. Quant. Spectrosc. Radiat.Transfer,11,1767(1971)

12/ Boercker D.B.,Iglesias C.A. and Dufty J.W.,Phys.Rev. A, 36,2254 (1987)

13/ Stamm R.,Talin B.,Pollock E.and Iglesias C.,Phys.Rev. A, 34,4144(1986)

14/ Stehlé C. and Feautrier N. ,J.Phys B: At. Mol. Phys. 17,1477,(1984)

15/ Stehlé C.,J.Quant.Spectrosc. Radiat. Transfer , to be published 46,(1990)

16/ Baranger M ,in "Atomic and Molecular Processes ",ed. by Bates D.R., Academic Press ,New-York,(1962)

17/ Feautrier N. and Tran-Minh N.,J.Phys B: At. Mol. Phys. 10,342,(1977)

18/ de Kertanguy A.,Tran Minh N. and Feautrier N., J.Phys B: At. Mol. Phys.12,365,(1979)

$19 /$ Seaton M,this issue

110/ Vidal C.R.,Cooper J. and Smith E.W., Astrophys. J. Suppl. Ser. 25,37,(1977)

111 Voslamber D.,Phys.Let. ,61A,27,(1977)

$112 /$ Lisitsa V.S. and Sholin G.V., Sov. Phys. JETP ,34,484,(1972)

$113 /$ Schöning T. and Buttler K. , Astron. Astrophys. Suppl. Ser ,to be publish.(1989)

114/ Greene R.L. and Cooper J. ,J.Quant.Spectrosc. Radiat. Transfer, 15,1025,(1975)

115/ Caby-Eyraud M.,Coulaud G. and Nguyen-Hoe ,J.Quant.Spectrosc. Radiat. Transfer ,15,593,(1975)

116/ Stehlé C.,Phys.Rev. A, 34,4153,(1986)

$117 /$ Stamm R.,Smith E.W. and Talin B.,Phys.Rev. A ,30,2039,(1984)

118/ Feautrier N.,Tran Minh N. and Van Regemorter H.,J.Phys B: At. Mol. Phys. 9,1871,(1976)

119 / Seidel J.,Z. Natürforsch.32a,1195,(1977)

120/ Oza D.H.,Greene R.L. and Kelleher D.E.,Phys.Rev. A ,38,2544,(1988) 